\title{
Morbilidad y mortalidad asociada a hospitalizaciones por influenza pandémica A (H1N1) 2009 en dos hospitales de la Región Metropolitana y evaluación de su impacto económico
}

\author{
Macarena Armstrong, Alberto Fica, Jeannette Dabanch, \\ Felipe Olivares, Rodrigo Fasce y Vjera Triantafilo
}

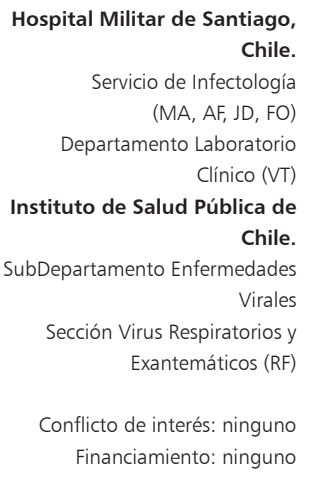

Recibido: 9 de agosto de 2012 Aceptado: 24 de octubre de 2012

Correspondencia a: Alberto Fica Cubillos albertofica@gmail.com

\author{
Morbidity and mortality associated to influenza A (H1N1) 2009 admissions in two \\ hospitals of the Metropolitan area and analysis of its economic impact
}

Influenza A (H1N1) 2009 infection was an important cause of morbidity and mortality in Chile. Aim: To characterize the clinical pattern of hospitalized patients, identify risk factors associated with ICU admission or death, and evaluate its economic impact. Patients and Methods: Twenty five adult patients admitted to 2 hospitals in the Metropolitan Area from May 2009 to December 2010 with PCR confirmed H1N1 infection were analyzed. Total hospital charges were obtained and, using data of registered cases, expenses for the whole country during the first epidemic wave were estimated. Results: All cases presented a risk factor: age over 60 years old $(\mathrm{n}=13$, $52 \%)$, co-morbid conditions $(n=24,96 \%)$ or pregnancy $(n=1,4 \%)$. Pneumonia was present in $64 \%(n=16)$ and $16 \%(\mathrm{n}=4)$ had hypotension. Only 6 patients $(24 \%)$ had a CURB-65 score $\geq 2$ and $36 \%(\mathrm{n}=9)$ requiring ICU admission. Case fatality rate was $16 \%(\mathrm{n}=4)$. By multivariate analysis, diabetes mellitus type 2 was independently associated with ICU admission or death (OR 8.12; $\left.\mathrm{IC}_{95} 1.11-59.2, \mathrm{p}<0.05\right)$. Hospital charges for those admitted to ICU or the intermediate care unit reached US\$ 20,304, and US\$ 1,262 for those admitted in general wards. We estimated US\$ 20 million in hospital charges for influenza related hospitalizations during the first wave for the whole country. Conclusions: A high proportion of patients affected by influenza A (H1N1) 2009 infection required ICU admission during 2009-2010. Case fatality rate associated to this infection was high, and diabetes mellitus type 2 was a risk factor for ICU admission or death. Hospital charges were higher for those admitted in critical care units and represented an important expenditure for Chile during the first wave. The CURB-65 score was inappropriate to recognize patients at risk of hospitalization or ICU admission.

Key words: Influenza A virus H1N1 subtype, pneumonia, Intensive Care Unit, diabetes mellitus type 2, hospital charges.

Palabras clave: Influenza A (H1N1), neumonía, Unidad de Terapia Intensiva, diabetes mellitus, gastos.

\section{Introducción}

L a emergencia de un nuevo tipo de influenza A (H1N1) en abril de 2009 por reordenamiento genético desencadenó una pandemia de vastas proporciones $^{1}$. Su llegada a Chile se registró a fines de abril del mismo año ${ }^{2}$ y durante los primeros cuatro meses se notificaron 340.000 casos de enfermedad tipo influenza, una tasa de ataque de $1,2 \%$ de la población, 1.585 hospitalizaciones y 130 fallecidos ${ }^{3}$. La experiencia nacional publicada hasta ahora ha sido escasa y restringida a pacientes atendidos en instituciones privadas de la Región Metropolitana (RM $)^{4,5}$ o en el extremo sur del país $^{2,6}$. Además, la información disponible sobre la carga económica asociada a las hospitalizaciones por esta enfermedad a nivel mundial es muy escasa e inexistente en Chile, lo que tiene relevancia en la adopción o evaluación de políticas preventivas. Los objetivos de este trabajo fue- ron efectuar una caracterización clínica de los pacientes hospitalizados por influenza pandémica A (H1N1) en dos hospitales institucionales de la RM durante los años 2009 y 2010 , identificar factores de riesgo involucrados en un desenlace grave o fatal y finalmente, determinar el impacto económico de esta enfermedad.

\section{Pacientes y Métodos}

Tipo de estudio e inclusión de casos: Diseño retrospectivo descriptivo. Los casos fueron detectados por los informes oficiales emanados desde el Instituto de Salud Pública informando sobre la presencia del virus influenza A (H1N1) 2009 pandémico desde mayo de 2009 a diciembre de 2010, luego de la derivación de muestras respiratorias para RPC en pacientes hospitalizados por sospecha de influenza. Se incluyeron los casos de dos 
hospitales de la Región Metropolitana, el Hospital Militar de Santiago y el Hospital de la Dirección Previsional de Carabineros. Ambos hospitales actúan como centros de referencia para los beneficiarios institucionales o sus cargas. Sólo se incluyeron pacientes $\geq 15$ años.

Características clínicas, evolución y desenlace: Los registros clínicos de estos pacientes fueron analizados con una plantilla estructurada para extraer información sobre variables demográficas, factores de riesgo conocidos para una evolución complicada, duración del período sintomático, manifestaciones clínicas, puntuación CURB-657 número de consultas previas al ingreso, tratamiento antiviral, evolución y condición al alta.

Definiciones: Se definió como baja saturación de $\mathrm{O}_{2}$ en la sangre, un valor $<90 \%$ en la oximetría de pulso digital y como hipotensión un valor de presión arterial sistólica $<90 \mathrm{mmHg}$ o una presión arterial diastólica $<60 \mathrm{mmHg}$ al ingreso. Para el reconocimiento de enfermedad tipo influenza se utilizó la definición actual vigente en Chile que incorpora fiebre y tos más alguna de los siguientes: odinofagia, mialgias o cefalea ${ }^{8}$. Se definió como desenlace grave el fallecimiento o el traslado a una unidad de mayor complejidad (unidad de cuidados intensivos o de tipo intermedio).

Gastos hospitalarios y estimación del gasto nacional: Sólo se incluyeron los casos identificados en el Hospital Militar de Santiago para esta parte del trabajo debido a la disponibilidad de datos específicos. Los gastos fueron separados por componentes, actualizados a precios de diciembre de 2010 utilizando la página del Instituto Nacional de Estadísticas de Chile (http://encina.ine.cl/ calculadoraipc/) y expresados en dólares americanos según la paridad cambiaria al 30 de diciembre de 2010 (1 US $\$=\$ 468,37$ pesos), dato obtenido del Banco Central de Chile. Para estimar el gasto nacional se tomaron en cuenta los casos hospitalizados hasta fines de agosto de 2009 que corresponden a la primera ola de la pandemia ${ }^{3}$. La proporción de pacientes que ingresó a unidades de pacientes críticos o salas generales fue tomada de los resultados de este mismo trabajo.

Análisis estadístico: Para el cálculo de los factores que pudiesen estar asociados a un desenlace grave (ingreso a UCI, UTI, conexión a VM o fallecimiento) se hizo el análisis mediante la prueba de Chi-cuadrado o test exacto de Fisher con un nivel de significación de 0,05. Para comparar las diferencias en los gastos hospitalarios se aplicó la prueba no paramétrica de Mann-Whitney. Finalmente, para establecer el valor predictor del sistema de puntuación CURB-65 para un desenlace fatal se hizo un análisis de curvas operador-receptor (COR).

\section{Resultados}

\section{Pacientes y condiciones de riesgo}

Un total de 25 pacientes hospitalizados por influenza pandémica $\mathrm{A}(\mathrm{H} 1 \mathrm{~N} 1)$ fueron identificados en los dos hospitales durante el período 2009-2010, 16 de ellos en el Hospital Militar de Santiago y 9 en el Hospital Previsional de Carabineros. La edad promedio fue de 57 años (rango 18-87 años) sin predominio por género. Ningún paciente estaba institucionalizado antes del ingreso y sólo uno de ellos postrado en su domicilio (Tabla 1).

Entre los factores de riesgo asociados a complicaciones de influenza, los predominantes en este grupo fueron la existencia de patología pulmonar $(40 \%)$ y diabetes mellitus (32\%). Otros factores se presentaron con menor frecuencia incluyendo cáncer $(16 \%)$, cardiopatía (16\%), hepatopatías ( $8 \%$ ) y condiciones neurológicas tales como eventos cerebrovasculares y demencia (12\%). Nuevos factores, tales como el alcoholismo y la obesidad mórbida también fueron identificados en esta serie en cifras superiores al $10 \%$. Aunque se observaron unos pocos casos

Tabla 1. Características generales de pacientes

hospitalizados por influenza A (H1N1) pandémica en dos hospitales de la RM, Chile 2009-2010

\begin{tabular}{|c|c|c|}
\hline Variable & & sultado \\
\hline Edad promedio en años (rango) & 57 & $(18-87)$ \\
\hline Edad $>60$ años & 13 & $(52 \%)$ \\
\hline Edad $>70$ años & 7 & $(28 \%)$ \\
\hline Género femenino n (\%) & 14 & (56) \\
\hline Postración n (\%) & 1 & (4) \\
\hline Tabaquismo previo $\mathrm{n}(\%)$ & 11 & (44) \\
\hline Tabaquismo actual n (\%) & 6 & (24) \\
\hline Asma / EPOC / fibrosis pulmonar $\mathrm{n}(\%)$ & 10 & $(40)$ \\
\hline Diabetes mellitus $n(\%)$ & 8 & (32) \\
\hline Cáncer n (\%) & 4 & (16) \\
\hline Alcoholismo n (\%) & 4 & (16) \\
\hline Cardiopatía n (\%) & 4 & (16) \\
\hline Obesidad mórbida n (\%) & 3 & (12) \\
\hline Evento cerebrovascular previo o demencia n (\%) & 3 & (12) \\
\hline Insuficiencia hepática o cirrosis n (\%) & 2 & (8) \\
\hline Inmunosupresión por corticoesteroides n (\%) & 2 & (8) \\
\hline Leucemia / linfoma / mieloma n (\%) & 1 & (4) \\
\hline VIH/SIDA n (\%) & 1 & (4) \\
\hline Embarazo n (\%) & 1 & (4) \\
\hline Cualquier condición de riesgo n (\%) & 25 & $(100)$ \\
\hline Una condición de riesgo (\%) & 14 & (56) \\
\hline Dos condiciones de riesgo (\%) & 4 & (16) \\
\hline Tres condiciones de riesgo (\%) & 7 & (28) \\
\hline
\end{tabular}


asociados a infección por VIH/SIDA, neoplasias oncohematológicas o inmunosupresión por corticoesteroides, no se registraron pacientes con epilepsia, enfermedad renal crónica, trasplante de órganos sólidos o de precursores hematopoyéticos en esta serie. El 100\% de los pacientes ingresados tenían un factor de riesgo conocido incluyendo un caso en una paciente con embarazo (Tabla 1).

\section{Distribución temporal}

Los casos incluidos en este trabajo se distribuyeron desde la semana epidemiológica 23 a la 36.

\section{Manifestaciones clínicas}

Las manifestaciones más frecuentes de fueron tos, fiebre, mialgias y disnea (Tabla 2), las que estuvieron presentes en al menos tres cuartos de los pacientes. En unos pocos casos se pesquisaron pacientes con alteraciones de conciencia y/o diarrea. La presencia de síntomas respiratorios altos fue baja en frecuencia. La presencia simultánea de fiebre y tos estuvo presente en $68 \%$ de los pacientes y la de enfermedad tipo influenza, sólo en $52 \%$ de los casos.

El $60 \%$ de los pacientes se presentó con bajos porcentajes de saturación (promedio 87,3\%, rango 60 a $99 \%$ ) y cerca de $30 \%$ con taquipnea $\geq 30 / \mathrm{min}$ (promedio 27,4 por min, rango $17-40$ por min). Dos tercios de los pacientes evidenciaron neumonía y cerca de $15 \%$ también tuvo hipotensión arterial al momento del ingreso (Tabla 2). La puntuación CURB-65 fue baja y sólo $24 \%$ tuvo valores $\geq 2$.

\section{Laboratorio}

El análisis de los parámetros de laboratorio revela que un tercio al menos se expresa con leucocitosis, neutrofilia, proteína $\mathrm{C}$ reactiva sobre $100 \mathrm{mg} / \mathrm{L}$ o hipoxemia (Tabla 3). Por otra parte, cerca de uno de cada cuatro o cinco pacientes presentó anemia, trombocitopenia o $\mathrm{LDH}$ plasmática muy elevada. En contraste, el aumento de la eritrosedimentación sobre $100 \mathrm{~mm} / \mathrm{h}$ fue infrecuente.

\section{Consultas previas, tratamiento antiviral y oportunidad del tratamiento}

Sólo $8 \%$ de los pacientes ( 2 de 24 con datos disponibles) se hospitalizó en la primera consulta y la mayor parte $(\mathrm{n}=16 ; 64 \%)$ con al menos una consulta previa. Seis pacientes $(24 \%)$ tuvieron al menos dos consultas previas al ingreso. Todos los pacientes salvo una excepción, recibieron tratamiento antiviral $(96 \%)$ y todos ellos con oseltamivir. El tratamiento se inició en promedio a los 3,5 días del inicio de los síntomas (rango 0 a 13 días), pero sólo $24 \%$ inicio tratamiento el primer día (6 de 24 tratados) y otro $33 \%$ el segundo o tercer día de evolución $(\mathrm{n}=8)$ para un porcentaje acumulado hasta el tercer día de $58 \%$. La fracción restante $(42 \% ; n=10)$ recibió tratamiento después de tercer día de iniciado los síntomas.

\section{Evolución y desenlace}

Un tercio de los pacientes debió ingresar a la Unidad de Cuidados Intensivos, todos ellos por falla respiratoria y adicionalmente, $28 \%$ de los pacientes requirió ser atendido en una unidad de cuidados intermedios. El total de pacientes que debió ingresar a una Unidad de Pacientes Críticos (ambos tipos de unidades), llegó a 64\% (Tabla 4).

Tabla 2. Manifestaciones clínicas de infección por influenza A (H1N1) pandémica en 25 pacientes hospitalizados

\begin{tabular}{lcc|} 
Variable & \multicolumn{2}{c}{ Resultado } \\
Tos & $\mathbf{n}$ & $\%$ \\
Fiebre & 23 & $(92)$ \\
Fiebre y tos & 19 & $(76)$ \\
Mialgias & 17 & $(68)$ \\
Disnea & 19 & $(76)$ \\
Expectoración & 19 & $(76)$ \\
Cefalea & 15 & $(60)$ \\
Rinorrea & 7 & $(28)$ \\
Odinofagia & 5 & $(20)$ \\
Enfermedad tipo influenza & 4 & $(16)$ \\
Compromiso de conciencia & 13 & $(52)$ \\
Diarrea & 3 & $(12)$ \\
Náuseas & 2 & $(8)$ \\
Saturación $\mathrm{O}_{2}$ ingreso $\leq 90 \%$ & 1 & $(4)$ \\
Frecuencia respiratoria $\geq 30 / m i n$ & 15 & $(60)$ \\
Hipotensión arterial & 7 & $(28)$ \\
CURB-65 & 4 & $(16)$ \\
Valor promedio & & \\
$0-1$ & 1 & \\
$\geq 2$ & 19 & $(76)$ \\
Neumonía & 6 & $(24)$ \\
\hline & 16 & $(64)$ \\
\hline
\end{tabular}

Tabla 3. Variables de laboratorio en pacientes ingresados por influenza A (H1N1) pandémica en dos hospitales de la RM, 2009-2010

\begin{tabular}{|lcc|} 
Parámetro & $\mathbf{n} / \mathrm{N}$ & $\%$ \\
Hemoglobina $<12 \mathrm{~g} / \mathrm{dL}$ & $6 / 24$ & 24 \\
Leucocitosis $>12.000$ céls $/ \mathrm{mm}^{3}$ & $9 / 25$ & 36 \\
Neutrofilia $>8.000$ céls $/ \mathrm{mm}^{3}$ & $10 / 24$ & 40 \\
Leucopenia $<4.000$ céls $/ \mathrm{mm}^{3}$ & $1 / 24$ & 4 \\
Linfopenia $<1.000$ céls $/ \mathrm{mm}^{3}$ & $12 / 24$ & 48 \\
Trombocitopenia $<150.000$ céls $/ \mathrm{mm}^{3}$ & $6 / 25$ & 24 \\
Eritrosedimentación $>100 \mathrm{~mm} / \mathrm{h}$ & $2 / 22$ & 8 \\
Proteína C reactiva $>100 \mathrm{mg} / \mathrm{L}$ & $9 / 24$ & 36 \\
Deshidrogenasa láctica $(\mathrm{LDH})>1.000 \mathrm{U} / \mathrm{L}$ & $5 / 23$ & 20 \\
PaO ${ }_{2}<60$ mmHg & $7 / 21$ & 28 \\
\hline
\end{tabular}


Cuatro pacientes fallecieron (16\%) y todos ellos el 2009 con una tasa de letalidad para ese año de 23,5\% (4 de 17 casos).

En la Tabla 5 se detallan las características de los pacientes fallecidos. Todos ellos presentaron neumonía con falla respiratoria. Tres de ellos ingresaron a UCI para VM y en un caso no se aplicó esta medida por limitaciones en el esfuerzo terapéutico. Las causas de muerte correspondieron a falla orgánica múltiple, SDRA, falla respiratoria hipoxémica y sobreinfección por $P$. aeruginosa, respectivamente.

Factores asociados a ingreso a UCI, conexión a VM (desenlace grave) o desenlace fatal

En el análisis univariado, el antecedente de DM tipo 2, la presencia de neumonía o de linfopenia, estuvieron asociados a ingreso a UCI o un desenlace fatal (Tabla 6). El análisis multivariado demostró que sólo el antecedente de DM tipo 2 se asoció en forma independiente y significativa a estos dos desenlaces combinados. Otras co-morbilidades, edad en diferentes categorías, saturación $<90 \%$, hipotensión arterial o diferentes resultados de laboratorio por categorías, no se asociaron a este resultado así como tampoco a diferentes ventanas de tiempo entre el inicio del tratamiento antiviral respecto al inicio de los síntomas o el número de consultas previas (datos no mostrados). La búsqueda de factores asociados a ingreso a UCI, conexión a VM o fallecimiento por separado no arrojó asociaciones significativas.

\section{Evaluación de sistema de puntuación CURB-65 para predecir mortalidad}

En la Figura 1 se muestra la capacidad de la puntuación CURB-65 para predecir mortalidad. El área bajo la curva fue 0,613 , sin diferencia significativa sobre la línea diagonal que indica la ausencia de un valor predictor $(\mathrm{p}>0,05)$. Un puntaje $\geq 2$ puntos tuvo una sensibilidad de $50 \%$ para identificar a los pacientes que fallecieron y $84 \%$ de especificidad.

\section{Estadía hospitalaria y aspectos económicos}

El promedio de estadía hospitalaria fue de 16,1 días (2 a 40 días) y de 402 días camas totales. La estadía hospitalaria promedio de los 9 pacientes que ingresaron a UCI en cualquiera de los dos hospitales fue de 26,1 días y de 10,4 días para aquellos que no ingresaron a UCI. Esta diferencia fue estadísticamente significativa $(p=0,004$ por prueba no paramétrica de Mann-Whitney). Una estadía > 8 días se asoció solamente con el ingreso a UCI con un valor OR 10,28 ( $\mathrm{IC}_{95}$ 1,03-102, $\left.\mathrm{p}<0,05\right)$. Los pacientes con asma e influenza tuvieron una tendencia a presentar una estadía menor pero las diferencias no fueron significativas (datos no mostrados). Los gastos hospitalarios se desglosan en la Tabla 7. El gasto total para los 16 pacientes alcanzó US\$ 210 mil y fue significativamente superior

\begin{tabular}{|c|c|c|}
\hline \multicolumn{3}{|c|}{$\begin{array}{c}\text { Tabla 4. Evolución y desenlace en } 25 \text { pacientes } \\
\text { hospitalizados por influenza A (H1N1) pandémica } \\
\text { en dos hospitales de la RM }\end{array}$} \\
\hline \multirow[t]{2}{*}{ Variable } & \multicolumn{2}{|c|}{ Resultado } \\
\hline & $\mathbf{n}$ & $\%$ \\
\hline \multicolumn{3}{|l|}{ Ingreso a Unidad de Pacientes Críticos (UPC) } \\
\hline Unidad de Cuidado Intensivos n (\%) & 9 & $(36)$ \\
\hline Unidad de Cuidados Intermedios n (\%)* & 7 & $(28)$ \\
\hline Subtotal UPC n (\%) & 16 & $(64)$ \\
\hline Ventilación mecánica invasora n (\%) & 9 & $(36)$ \\
\hline Fallecidos n (\%) & 4 & $(16)$ \\
\hline \multicolumn{3}{|c|}{$\begin{array}{l}\text { *Excluye aquellos que estuvieron en Unidad de Cuidados Intensivos } \\
\text { antes o después de Unidad Intermedios. }\end{array}$} \\
\hline
\end{tabular}

Tabla 5. Características de pacientes fallecidos durante hospitalización por influenza A (H1N1) pandémica

\begin{tabular}{|c|c|c|c|}
\hline Caso & $\begin{array}{l}\text { Edad (años), } \\
\text { sexo }\end{array}$ & Condiciones de riesgo & Evolución \\
\hline 1 & $69, \mathrm{~F}$ & Diabetes mellitus tipo 2 & $\begin{array}{l}\text { Neumonía multilobar } \\
\text { Falla respiratoria que requiere VM en UCl } \\
\text { Neumonía asociada a VM y bacteriemia no } \\
\text { asociada a CVC } \\
\text { Falla orgánica múltiple } \\
\text { Fallece a } 30 \text { días del ingreso }\end{array}$ \\
\hline 2 & $65, F$ & $\begin{array}{l}\text { Leucemia linfática crónica } \\
\text { Tabaquismo }\end{array}$ & $\begin{array}{l}\text { Neumonía } \\
\text { Falla respiratoria que requiere VM en UCI } \\
\text { SDRA y falla respiratoria refractaria } \\
\text { Fallece a los } 11 \text { días del ingreso }\end{array}$ \\
\hline 3 & $64, M$ & $\begin{array}{l}\text { Fibrosis pulmonar } \\
\text { Diabetes mellitus tipo } 2\end{array}$ & $\begin{array}{l}\text { Neumonía } \\
\text { Falla respiratoria hipoxémica refractaria } \\
\text { Se limita el esfuerzo terapéutico } \\
\text { Manejo en Unidad Intermedia sin VM } \\
\text { Fallece a los } 9 \text { día del ingreso }\end{array}$ \\
\hline 4 & $34, M$ & $\begin{array}{l}\text { Cáncer en el SNC } \\
\text { Inmunosupresión por } \\
\text { corticoesteroides }\end{array}$ & $\begin{array}{l}\text { Neumonía } \\
\text { Falla respiratoria que requiere } \mathrm{VM} \text { en } \mathrm{UCl} \\
\text { Fallece por sepsis por sobreinfección pulmonar } \\
\text { con neumonía cavitada por } P \text {. aeruginosa con } \\
\text { bacteriemia } \\
\text { Fallece a los } 35 \text { días del ingreso }\end{array}$ \\
\hline
\end{tabular}

Tabla 6. Factores asociados con ingreso a UCI o desenlace fatal. Análisis univariado y multivariado

\begin{tabular}{|lccc|}
\hline Factor & OR & IC95 & p \\
Análisis univariado & & & \\
Diabetes mellitus tipo 2 & 6,5 & $0,937-45,1$ & 0,05 \\
Neumonía & 10,28 & $1,03-102,7$ & $<0,05$ \\
Linfopenia $<1.000$ céls/mm ${ }^{3}$ & 7,0 & $1,04-46,94$ & $<0,05$ \\
Análisis multivariado & & & \\
Diabetes mellitus tipo 2 & 8,12 & $1,11-59,2$ & $<0,05$ \\
\hline
\end{tabular}


Tabla 7. Gastos asociados a hospitalización por influenza A (H1N1) pdm en 16 pacientes en un hospital de la Región Metropolitana, 2009-2010

\begin{tabular}{|c|c|c|c|c|c|c|c|}
\hline \multirow[t]{2}{*}{ Parámetro } & \multicolumn{2}{|c|}{$\begin{array}{l}\text { Gasto en pacientes que ingresaron a UPC } \\
\qquad n=10\end{array}$} & \multicolumn{2}{|c|}{$\begin{array}{l}\text { Gasto en pacientes que no ingresaron a UPC } \\
\qquad n=6\end{array}$} & \multicolumn{3}{|c|}{ Gasto global } \\
\hline & Pesos chilenos & US\$ & Pesos chilenos & US\$ & Pesos chilenos & US\$ & $\%$ \\
\hline Estadía hospitalaria & 44.184 .114 & $94,336^{*}$ & 1.743 .109 & 3,722 * & 45.927 .223 & 98,058 & 47 \\
\hline Laboratorio & 5.613 .490 & $11,985^{*}$ & 471.240 & $1,006^{*}$ & 6.084 .730 & 12,991 & 6 \\
\hline Imágenes & 1.456 .705 & 3,110 & 245.863 & 525 & 1.702 .568 & 3,635 & 2 \\
\hline Fármacos & 26.722 .519 & $57,054^{*}$ & 467.828 & 999* & 27.190 .347 & 58,053 & 28 \\
\hline Insumos & 10.689 .572 & $22,823^{*}$ & 270.670 & $578^{*}$ & 10.960 .242 & 23,401 & 11 \\
\hline Procedimientos & 1.457 .470 & 3,112 & 3.388 & 7 & 1.460 .858 & 3,119 & 1 \\
\hline Otros & 7.736 .065 & $16,517^{*}$ & 416.918 & $890 *$ & 8.152 .983 & 17,407 & 8 \\
\hline Total & 95.098 .197 & $203,041 *$ & 3.546 .098 & 7,571 * & 98.644 .295 & 210,612 & 100 \\
\hline Gasto por paciente & & 20,304 & & 1,262 & & 13,163 & \\
\hline
\end{tabular}

Figura 1. Curva operador receptor para puntuación CURB-65 en la predicción de mortalidad.

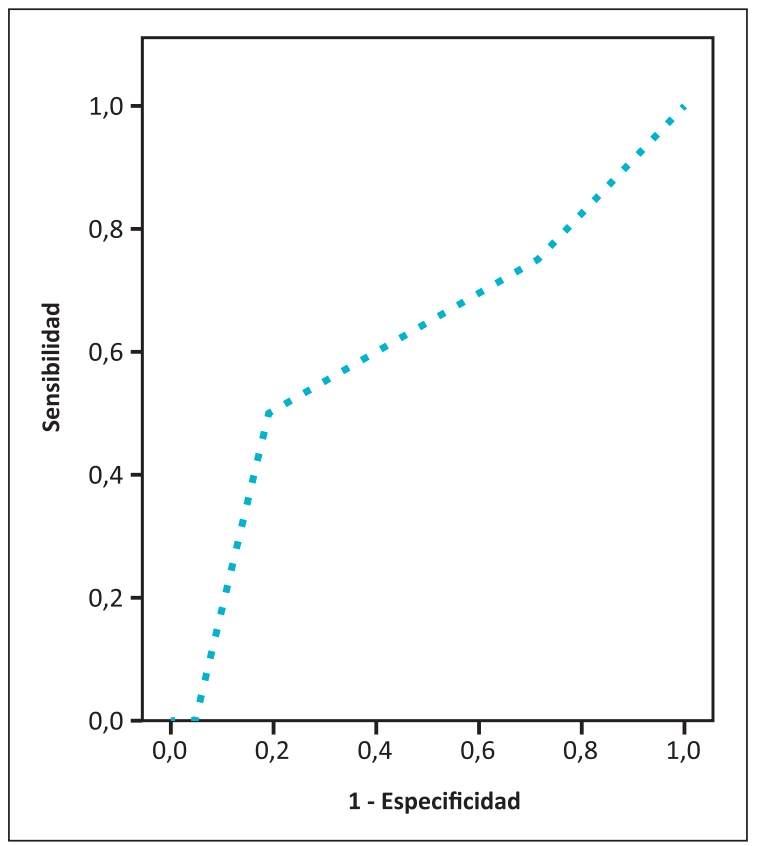

en los pacientes ingresados a la Unidad de Pacientes Críticos (UCI e intermedio) respecto al grupo de pacientes manejados en sala (US\$ 203 mil versus 7.500, prueba no paramétrica de Mann-Whitney $\mathrm{p}<0,05)$. El análisis por componentes indicó que los gastos fueron más altos en estadía hospitalaria, laboratorio, fármacos, insumos y gastos varios pero no en procedimientos invasores o quirúrgicos. En términos generales, la mitad del gasto estuvo asociado a la estadía hospitalaria. El gasto por paciente en aquellos que ingresaron a la UPC fue $\sim$ US\$ 20,000 y US\$ 1,200 en el grupo que no fue admitido en este tipo de unidades en el Hospital Militar.

\section{Estimación de gasto en primera ola de influenza}

Para la estimación de los gastos hospitalarios en todo Chile en la primera ola se asumió que $40 \%$ de los pacientes reportados como hospitalizados entre mayo y agosto de 2009 ingresaron a salas generales $(n=634)$ y que el $60 \%$ restante lo hizo en Unidades de Pacientes Críticos (UCI e Intermedios, $\mathrm{n}=951$ casos). Los datos de las proporciones fueron sacados de este trabajo y el número de casos durante la primera ola, de una publicación nacional que da cuenta de ello ${ }^{3}$. Ello determina un gasto estimado de US\$ 800.017 para el subgrupo manejado en sala y de US\$ 19.309.175 para el subgrupo en Unidades Intensivas e Intermedias, respectivamente. De esta manera, los gastos hospitalarios globales hasta comienzos de agosto de 2009 fueron estimados en US\$20.109.193 para todo el país.

\section{Discusión}

\section{Factores de riesgo}

En este trabajo, 25 casos de influenza pandémica A (H1N1) fueron identificados en dos hospitales de la RM durante dos años consecutivos. Todos los casos estuvieron asociados a co-morbilidad o condiciones de riesgo para complicaciones de influenza. La existencia predominante de pacientes con patología pulmonar crónica, diabetes mellitus o cáncer es concordante con datos nacionales ya publicados ${ }^{4}$ y se pudo observar la presencia de grupos de riesgo descritos recientemente como la obesidad mórbida 9 .

Estos factores aumentan ya sea el riesgo de hospitalización, ingreso a UCI o muerte. En nuestro trabajo se pudo demostrar que el antecedente de diabetes mellitus tipo 2 aumenta significativamente el riesgo de ingreso a UCI o muerte tal como ha sido reportado en revisiones sistemáticas recientes ${ }^{9,10}$. Otras asociaciones no pudieron ser demostradas por el reducido tamaño de la población 
bajo estudio. Durante el primer año de la pandemia, 1.585 personas se hospitalizaron en Chile por influenza y $130 \mathrm{de}$ ellas fallecieron (letalidad hospitalaria $8,2 \%)^{3}$. Utilizando esta base de datos más amplia, Dabanch et al, pudieron identificar adicionalmente otros factores asociados a un desenlace fatal como la edad mayor a 60 años o la portación de alguna co-morbilidad en la población entre 15 y 49 años $^{3}$. En nuestra serie, la causa de muerte fue variada e incluyo falla respiratoria refractaria, falla orgánica múltiple o sepsis nosocomial, que representa un perfil compatible con lo observado en otras latitudes. ${ }^{11}$ De esta manera, los factores de riesgo para hospitalización, ingreso a UCI o muerte, han resultado esencialmente similares a los de la influenza A estacional antes de la pandemia, a los que se ha sumado la obesidad mórbida ${ }^{9}$ pero no han corroborado un cambio mayor de virulencia expresado en desenlaces fatales en la población joven sin co-morbilidad.

\section{Características clínicas, sistemas de puntuación y laboratorio}

Los pacientes que se hospitalizan por influenza no parecen presentarse en forma predominante con una enfermedad tipo influenza, limitando la sospecha clínica inicial. Además, 16\% de los pacientes presentó hipotensión arterial al ingreso, un elemento clínico no asignado históricamente a pacientes con infección pulmonar de origen viral, excepto en el caso de pacientes con infección por hantavirus ${ }^{12}$. El shock hemodinámico fue detectado en 3 de 99 pacientes $(\sim 3 \%)$ que ingresaron a una clínica privada de la RM el primer año de la pandemia ${ }^{5}$, en $20 \%$ de 20 hospitalizados en el sur de Chile y en grandes series de pacientes con influenza A (H1N1) 2009 se han reportado cifras de shock hemodinámico entre 1,7 y $8,8 \%$ de la población bajo estudio ${ }^{2,13,14}$. Ello genera imperfecciones del sistema de puntuación CURB-65 para reconocer casos graves por influenza. El valor promedio de este puntaje fue notoriamente bajo con un valor promedio de 1 , similar a lo observado por otros autores para influenza A (H1N1) $2009(0,65)^{13}$. Ello ocurre por dos razones opuestas: el sistema CURB-65 considera la hipotensión arterial y el compromiso de conciencia, que si bien están presentes en estos pacientes, no corresponden a un rasgo frecuente, y por otras parte, no incluye la baja saturación de $\mathrm{O}_{2}$ a no ser que se acompañe de taquipnea. De esta manera, la nueva influenza A (H1N1) pandémica, pudo haber golpeado las unidades de emergencia provocando un rebote de pacientes debido a que no presentaron criterios de gravedad habituales. En esta serie, sólo 8\% se hospitalizó en la primera consulta. Esta situación motivó que en los sistemas de vigilancia postpandemia definidos por la OPS se hayan considerado nuevos criterios de gravedad que están basados ahora en la presencia de fiebre y síntomas respiratorios junto a taquipnea ( $\geq 30 / \mathrm{min}$ ) o una baja saturación de oxígeno por oximetría de pulso digital con $\mathrm{O}_{2}$ ambiental $(<90 \%)$ y no en el compromiso de conciencia o hipotensión arterial como se utiliza en el sistema de puntuación CURB- $65^{15}$. De hecho, la taquipnea es el único marcador aplicado en ambos sistemas. Ello requiere una pronta actualización de los criterios de gravedad aplicados en adultos en Chile afectados por neumonía adquirida en la comunidad según se desprende de lo ya comentado. Desde la perspectiva del laboratorio, la trombocitopenia y el aumento en los valores de $\mathrm{LDH}$, presentes entre 20 y $24 \%$ de estos casos, también han sido descritos en otras series, incluso con frecuencias más elevadas (42\% y valores promedio de 744-945 U/L, respectivamente) y asociados a mayor gravedad, extensión del compromiso pulmonar, riesgo de ingreso a UCI o muerte ${ }^{11,13,16,17}$. Estas alteraciones de laboratorio cuestionan la supuesta distinción entre infecciones virales y bacterianas ${ }^{18}$ y están en línea con el hallazgo de leucocitosis, neutrofilia y valores elevados de PCR en estos pacientes, los que también han sido descritos en otras series de pacientes con influenza A (H1N1) 200914,17,19.

En las series reportadas, la mayor parte de los pacientes que se hospitalizan por influenza A(H1N1) 2009 tuvieron neumonía, con cifras variables entre 30 y $100 \%$ y que incluyen la cifra observada en nuestra serie ${ }^{4,13,17,19}$. La fracción remanente, se explica por descompensaciones de patologías de base, mujeres embarazadas ${ }^{5}$ o complicaciones infrecuentes como las de tipo neurológico, estas últimas no observadas en este reporte ${ }^{20}$.

Cerca de dos tercios de los pacientes en esta serie ingresaron a una sala de mayor complejidad y un tercio a UCI, grupo que requirió conexión a VM por falla respiratoria. La proporción de pacientes que ingresa a UCI ha sido variable en la literatura médica con rangos de 22,6 a $43 \%{ }^{14,17}$ y de 12,8 a $27,7 \%$ para conexión a $\mathrm{VM}^{11,14}$. No obstante esta dispersión, las tasas de letalidad hospitalaria observadas en este trabajo (16\%) están en línea con lo reportado por otros autores $(15 \text { a } 17 \%)^{11,17}$ pero difieren con la ausencia de casos letales observados en dos clínicas privadas en la RM de Chile ${ }^{4,5}$, lo que en al menos un caso es explicado por el bajo perfil de co-morbilidad y gravedad incluido en la serie ${ }^{5}$.

Casi todos los pacientes recibieron tratamiento con oseltamivir en rangos de tiempo variables respecto al inicio de los síntomas, pero su retraso no estuvo asociado al ingreso a UCI o un desenlace fatal. Diversos autores han explorado la importancia de este aspecto y no han logrado mayoritariamente encontrar una asociación con la gravedad de la evolución del cuadro de influenza o un desenlace fatal ${ }^{11,13,16,21}$. No obstante, una serie española con más de 500 pacientes adultos logró demostrar que el tratamiento antiviral hasta las $72 \mathrm{~h}$ del inicio de los síntomas, tuvo un efecto protector sobre ingreso a UCI o muerte (OR 0,32) 22. En forma adicional, algunos autores han podido demostrar que el mayor riesgo de ingreso a 
UCI o muerte se asocia al ingreso después del segundo día o cuarto día de evolución o a una condición rural 2,3,21,23.

Gastos hospitalarios y estimación del gasto nacional en la primera ola

La información sobre los gastos hospitalarios asociados a los ingresos por influenza A(H1N1) 2009 es escasa a la fecha y sólo se dispone de datos asociados a pacientes pediátricos en E.U.A. ${ }^{24}$. El gasto promedio por paciente ingresado a UCI pediátrica fue de US\$ 254 mil versus US\$ 20 mil en pacientes adultos en UCI y de US\$ 14 mil para los pacientes atendidos en sala versus los US\$1,2 mil observados en nuestro centro hospitalario, respectivamente. La estimación del gasto nacional efectuada en este trabajo da cuenta de la magnitud económica de la pandemia en su primera ola y sólo considera la faceta hospitalaria. Es probable que los 20 millones de dólares estimados estén en el límite inferior del gasto real debido a la participación de clínicas privadas con facturaciones mayores a las que se generan en los hospitales públicos $\mathrm{o}$ institucionales.

Los hallazgos de este trabajo están limitados por la naturaleza retrospectiva que pudo haber impedido extraer información relevante por subregistro clínico y por el bajo poder estadístico a pesar de la inclusión de dos hospitales para corregir este impedimento. Además, la circulación de infuenza A (H1N1) prácticamente desapareció después de 2010 impidiendo ampliar la ventana temporal del estudio. Por otra parte, la co-infección inicial no fue estudiada sistemáticamente y es posible que haya ocurrido más allá de los pocos casos identificados. Finalmente, la estimación de los gastos hospitalarios está basada en la facturación generada en un hospital de tipo público con un número reducido de casos y no se encontraron datos sobre la tasa de hospitalización en unidades intermedias para complementar aquella existente sobre unidades críticas y así confirmar que $60 \%$ en la suma de ambos tipos de unidades utilizado en este trabajo, es extrapolable.

\section{Conclusiones}

En esta serie de 25 pacientes hospitalizados por influenza pandémica A (H1N1) 2009 en dos años consecutivos se pudo demostrar que todos ellos tenían una condición de riesgo. Los pacientes se presentaron sólo en la mitad de los casos con una enfermedad tipo influenza, en $60 \%$ con una baja saturación en la oximetría de pulso digital y en aproximadamente $30 \%$ con taquipnea $\geq 30 /$ min. Al menos dos tercios de ellos presentaron neumonía y uno de cada 7 pacientes tuvo hipotensión arterial asociada. La relativa baja frecuencia del compromiso hemodinámico o del compromiso de conciencia explica que la puntuación CURB-65 haya sido baja y que no sea útil para reconocer el riesgo de muerte en estos pacientes y ello justifica la adopción reciente de sistemas de vigilancia basados predominantemente en la conjunción de síntomas respiratorios con elementos de falla respiratoria como la taquipnea y/o una baja saturación de $\mathrm{O}_{2}$. No obstante, es necesario actualizar los criterios de gravedad vigentes en las guías nacionales para casos con neumonía adquirida en la comunidad, basados hasta ahora en el sistema de puntuación CURB-65 que como vimos es poco sensible para cuadros graves de influenza. Desde el punto de vista del laboratorio, la presencia de leucocitosis en $36 \%$ y de neutrofilia en $40 \%$ de los casos al ingreso, subraya que no hay distinciones fáciles entre patógenos bacterianos y virales en pacientes que se hospitalizan por neumonía, hecho que queda además demostrado por la presencia de trombocitopenia y $\mathrm{LDH}$ elevada $>1.000 \mathrm{U} / \mathrm{L}$ en aproximadamente uno de cada 5 casos con influenza A (H1N1) pandémica. La relevancia y agresividad clínica de la emergencia de un nuevo tipo viral por reordenamiento genético queda demostrada por la alta tasa de ingreso a la Unidad de Cuidados Intensivos que alcanzó un tercio de los pacientes hospitalizados y por la necesidad conjunta de una sala de Cuidados Intensivos o de tipo intermedio en dos tercios de los casos. A pesar de que en esta serie sólo 4 pacientes fallecieron, ello representa una letalidad de un $16 \%$. Se puso demostrar que la presencia de diabetes mellitus tipo 2 se asoció en forma significativa e independiente al ingreso a UCI o a un desenlace fatal y también se observó que la estadía promedio de estos pacientes superó las dos semanas. Finalmente, los gastos hospitalarios fueron considerables, especialmente en el subgrupo que ingresó a UCI o Intermedio y se pudo estimar un gasto mínimo nacional de 20 millones de dólares por concepto de hospitalización para los primeros 4 meses de la pandemia en Chile el 2009.

Agradecimientos. Los autores de este trabajo desean agradecer la valiosa cooperación de los integrantes de la Unidad de Bioestadística del Hospital Militar y del Laboratorio Clínico.

\section{Resumen}

Introducción: La infección por influenza A (H1N1) pandémica representó una importante carga de morbilidad y mortalidad en Chile. Objetivo: Caracterización clínica de pacientes hospitalizados durante los años 2009 y 2010 , identificar factores de riesgo asociados con ingreso a UCI o muerte y determinar el impacto económico de esta enfermedad. Pacientes y Métodos: Análisis de las características clínicas y evolución en un grupo de 25 pacientes adultos ingresados a dos hospitales institucionales en la Región Metropolitana confirmados por RPC desde mayo de 2009 a diciembre de 2010. Estudio de gastos hospitalarios y estimación de gasto nacional según registro de 
casos atendidos desde mayo a agosto de 2009. Resultados. Todos los pacientes presentaron una condición de riesgo: edad $>60$ años (n: 13, 52\%), co-morbilidad (n: 24, 96\%) o embarazo ((n: 1, 4\%). El 64\% (n: 16) presentó neumonía y $16 \%$ tuvieron hipotensión arterial (n: 4). Sólo 6 pacientes (24\%) tuvieron puntuación CURB-65 $\geq 2$. Un 36\% (n: 9) requirió manejo en Unidad de Cuidados Intensivos (UCI) y 4 pacientes fallecieron (16\%). Por análisis multivariado, el antecedente de diabetes mellitus tipo 2 se asoció en forma significativa e independiente al ingreso a UCI o a un desenlace fatal (OR 8,12; $\left.\mathrm{IC}_{95} 1,11-59,2, \mathrm{p}<0,05\right)$. El gasto por paciente en aquellos que ingresaron a la UCI o Intermedio alcanzó los US\$20.304 y US\$1.262, para los que no ingresaron a estas unidades. Para Chile, se estimó un gasto mínimo de 20 millones de dólares por concepto de hospitalización para los primeros cuatro meses de la pandemia el 2009, asumiendo que $60 \%$ ingresó a UCI o Unidades Intermedias. Conclusiones: Una alta proporción de los pacientes afectados por influenza A (H1N1) 2009 requirió ingreso a UCI durante los años 2009-2010. La letalidad de esta infección fue elevada y la diabetes mellitus tipo 2 fue un factor de riesgo para ingreso a UCI o muerte. Los gastos hospitalarios fueron elevados, especialmente en los que ingresaron a unidades críticas. El sistema CURB-65 tiene una baja capacidad para reconocer riesgo de hospitalización o muerte en estos pacientes.

\section{Referencias bibliográficas}

1.- Novel Swine-Origin Influenza A (H1N1) Virus Investigation Team. Emergence of a novel swine-origin influenza $\mathrm{A}(\mathrm{H} 1 \mathrm{~N} 1)$ virus in humans. N Eng J Med 2009; 361: 1-10.

1a.- Nickel K B, Marsden-Haug N, Lofy K H, Turnberg W L, Rietberg K, Lloyd J K, et al. Age as an independent risk factor for Intensive Care Unit admission or death due to 2009 pandemic influenza A (H1N1) virus infection. Public Health Rep 2011; 126: 349-53.

2.- Chilean Task Force for study of Pandemic Influenza A, Pedroni E, García M, Espínola V, Guerrero A, González C, et al. Outbreak of 2009 pandemic influenza A (H1N1), Los Lagos, Chile, April-June 2009. Euro Surveill 2010; 15 (1). pii: 19456.

3.- Dabanch J, Perret $C$, Nájera M, González $C$, Guerrero A, Olea A, et al. Age as risk factor for death from pandemic (H1N1) 2009, Chile. Emerg Infect Dis 2011; 17: 1256-58.

4.- Rabagliati R, Siri L, Pérez C, Labarca J, Ferrés M. Influenza pandémica A (H1N1) 2009: epidemiología, características clínicas y diferencias con influenza estacional en Chile. Rev Chilena Infectol 2011; 28: 546-53.

5.- Torres JP, O'Ryan M, Herve B, Espinoza R, Acuña $G$, Mañalich J, et al. Impact of the novel influenza A (H1N1) during the autumnwinter season in a large hospital setting in Santiago, Chile. Clin Infect Dis 2010; 50: 860-8.

6.- Riquelme R, Riquelme $\mathrm{O}$, Rioseco $\mathrm{M} \mathrm{L}$, Inzunza $\mathrm{C}$, Contreras $\mathrm{C}$, Gómez $\mathrm{Y}$, et al. Impacto clínico de la influenza A H1N1 pandémica en el Hospital de Puerto Montt, Chile. Rev Med Chile 2011; 139: 321-6.

7.- Lim W S, Van der Eerden M M, Laing R, Boersma W G, Karalus N, Town G I, et al. Defining community acquired pneumonia severity on presentation to hospital: an international derivation and validation study. Thorax 2003; 58: 377-82.

8.- Ministerio de Salud de Chile. Guía de Vigilancia intensificada de las infecciones respiratorias agudas graves. Julio 2011. Disponible en http:// epi.minsal.cl/epi/html/bolets/reportes/Influenza/ Guia_de_Vigilancia_Intensificaca_de_las_IRA_ junio2011.pdf

9.- Van Kerkhove M D, Vandemaele K A H, Shinde V, Jaramillo-Gutiérrez G, Koukounari A, Donnelly C A, et al. Risk factors for severe outcomes following 2009 influenza A (H1N1) infection: A global pooled analysis. PLoS Med 2011; 8 (7): e1001053

10.- Allard R, Leclerc P, Tremblay C, Tannembaum T N. Diabetes and the severity of pandemic influenza A (H1N1) infection. Diabetes Care 2010; 33: 1491-93.

11.- Xi X, Xu Y, Jiang L, Li A, Duan J, Du B, et al. Hospitalized adult patients with 2009 influenza A (H1N1) in Beijing, China: risk factors for hospital mortality. BMC Infect Dis 2010; 10:256

12.- Sotomayor V, Olea A, Labraña M, Castillo C, Ortega C, Riquelme R, et al. Diagnóstico y manejo del síndrome cardiopulmonar por hantavirus. Chile-2007. Rev Chilena Infectol 2009; 26: 68-86.

13.- Cho W H, Kim Y S, Jeon D S, Kim J E, Kim K I, Seol H Y, et al. Outcome of pandemic H1N1 pneumonia: Clinical and radiological findings for severity assessment. Korean J Intern Med 2011; 26: 160-7.

14.- Viasus D, Cordero E, Rodríguez-Baño J, Oteo JA, Fernández-Navarro A, Ortega L, et al. Changes in epidemiology, clinical features and severity of influenza A (H1N1) 2009 pneumonia in the first post-pandemic influenza season. Clin Microbiol Infect 2012; 18:E55-E62.

15.- Ministerio de Salud de Chile. Guía para el diagnóstico y manejo de casos de influenza. 2012. Disponible en http:// www.minsal.gob.cl/portal/url/item/ bbb1fd555fca7720e0400101650149c0.pdf

16.- Ríos F G, Estenssoro E, Villarejo F, Valentini R, Aguilar L, Pezzola D, et al. Lung function and organ dysfunctions in 178 patients requiring mechanical ventilation during the 2009 influenza A (H1N1) pandemic. Crit Care 2011;
15: R201.

17.- Tabarsi P, Moradi M, Baghaei P, Hashemian S M, Nadji SA, Fakharian A, et al. Factors associated with death or intensive care unit admission due to pandemic 2009 influenza A (H1N1) infection. Ann Thorac Med 2011; 6: 91-5.

18.- Fiumefreddo R, Zaborsky R, Haeuptle J, Christ-Crain M, Trampuj A, Steffen I, et al. Clinical predictors for Legionella in patients presenting with community-acquired pneumonia to the emergency department. BMC Pulmonary Medicine 2009; 9: 4.

19.- Sertogullarindan B, Ozbay B, Gunini H, Sunnetcioglu A, Arisoy A, Bilgin H M, et al. Clinical and prognostic features of patients with pandemic 2009 influenza A (H1N1) virus in the intensive care unit. Afr Health Sci 2011; 11: $163-70$

20.- Bustos B R, Andrade Y F. Encefalopatía aguda y muerte cerebral, en un niño con influenza A (H1N1) 2009 durante la primera ola pandémica. Rev Chilena Infectol 2010; 27: 413-6.

21.- Kirakli C, Tatar D, Cinem P, Epidoglu O, Coskun M, Celikten E, et al. Survival from severe pandemic H1N1 in rural and urban Turkey: A case series. Respir Care 2011; 56: 790-5.

22.- Viasus D, Paño-Pardo J R, Pachón J, Campins A, López-Medrano F, Villoslada A, et al. Factors associated with severe disease in hospitalized adults with pandemic (H1N1) 2009 in Spain. Clin Microbiol Infect 2011; 17: 738-46.

23.- Nickel K B, Marsden-Haus N, Lofy K H, Turnberg W L, Rietberg K, Lloyd J K, et al. Age as an independent risk factor for intensive care unit admission or death due to 2009 pandemic influenza A (H1N1) virus infection. Publ Health Rep 2011; 126: 349-53.

24.- Kumar S, Havens P L, Chusid M J, Willoughby Jr R E, Simpson P, Henrickson K J. Clinical and epidemiological characteristics of children hospitalized with 2009 H1N1 pandemic influenza A infection. Ped Infect Dis J 2010; 29: 591-4. 